ISSN 00156043

\title{
EDITORIAL
}

\section{El hambre, ¿tsunami silencioso?}

\section{Consejo de Redacción'}

Palabras clave: Hambre, seguridad alimentaria, políticas agrícolas, precio de alimentos.

Key words: Famine, food security, agricultural policies, food price.

Se acabó el mundo de los alimentos baratos. Con suerte y buenas políticas, podremos alcanzar un nuevo equilibrio ${ }^{2}$.

A pesar de todos los avances en el terreno social, político, económico y tecnológico logrados por la civilización, nuestro mundo sigue conviviendo con el hambre ${ }^{3}$.

El hambre es una de las manifestaciones más extremas de la marginalización y la pobreza, y constituye una de las más flagrantes violaciones de los Derechos Humanos" 4.

\footnotetext{
1 Texto cerrado a mediados de octubre de 2008. Los cambios en el panorama económico y financiero internacional se suceden con ritmo vertiginoso. Hemos mantenido, sin embargo, nuestro texto por tratar de un problema realmente estructural.

2 The Editors (2008), "The silent tsunami", The Economist, 17 de abril.

${ }^{3}$ Ministerio de Asuntos Exteriores y Cooperación, Secretaría de Estado de Cooperación Internacional, DIrección General de Planificación y Evaluación de Polítcas para el Desarrollo (2007), Estrategia de lucha contra el Hambre de la Cooperación Española, Madrid. El autor del documento base de esta estrategia es Enrique de LOMA Osorio. Cf. Prólogo.

${ }^{4}$ Ibidem., p. 9.
} 


\section{Presentación}

El título no es nuestro. Según parece, el recurso a la metáfora del tsunami, utilizado hasta la saciedad, partió del Programa Mundial de la Alimentación (PMA: World Food Programme), la agencia de Naciones Unidas que es la más importante institución mundial de distribución de alimentos ${ }^{5}$.

En los últimos meses de 2007 y primera mitad de 2008 se desató la alarma mundial por el rápido ascenso de los precios de los productos alimenticios, con efectos particularmente devastadores para los países pobres. Los hechos eran claros: según la FAO (Food and Agriculture Organization), los precios de mercado de los cereales destinados a los países menos avanzados (PMA) aumentaron un 37\% entre 2006 y 2007 y un $56 \%$ entre 2007 y $2008^{6}$.

Y lo peor es que las previsiones no son optimistas. El 29 de mayo de 2008, unos días antes de que la FAO inaugurara en Roma una cumbre dedicada al tema "Seguridad alimentaria mundial: los desafíos del cambio climático y de la bioenergía", dicha organización y la Organización para la Cooperación y el Desarrollo Económico (OCDE) presentaron un informe acerca de las subidas recientes de los precios de los alimentos; en él aseguraban que dichos precios seguirán elevados durante la próxima década, aunque los "records" de estos meses pasados no perduren. Así, calculan que productos básicos como los cereales o el arroz podrían subir entre un 60 o un $80 \%$ en este periodo, niveles sustancialmente superiores a los que han prevalecido durante los pasados diez años. La evolución de los precios en julio y agosto parece haber concedido un respiro a las fuertes subidas; tanto el maíz como el trigo y el arroz han bajado de forma sustancial.

Más allá de sus características coyunturales, la urgencia y la gravedad del tema nos han movido a tomarlo como objeto de este artículo editorial, aprovechando la avalancha de publicaciones de estos meses. Al examinarlas, uno percibe desde el primer momento que estamos ante una cuestión compleja y controvertida. Nosotros no queremos eludir esa complejidad con simplificaciones peligrosas, que suelen desembocar en opiniones muy encontradas donde no es fácil huir de los

${ }^{5}$ Lo encontramos, por ejemplo, en sendos artículos aparecidos casi al mismo tiempo, con exactamente el mismo título: el citado en la nota 2 y el de America, The National Catholic Weekly, 19 de mayo de 2008.

${ }^{6}$ Citado por C. Malagol (2008), "Prezzi del cibo e fame nel mondo", Aggiornamenti Sociali, n. 07-08, 493-500, cf. p. 493. Usaremos ampliamente esta excelente síntesis de la revista homóloga a la nuestra, de los jesuitas italianos. 
dogmatismos. Por eso hemos optado por intentar responder sucesivamente a las siguientes cuestiones: ¿qué está pasando? ¿¿cuáles son las causas de estas subidas de precios? ¿̇desde qué presupuestos éticos debemos abordar la cuestión? ¿qué medidas a corto plazo se sugieren? ¿cuáles son las acciones a emprender a medio y largo plazo?

\section{2. ¿Qué está pasando? ¿tsunami o plaga endémica?}

La metáfora del tsunami, tan utilizada para referirse a la "nueva" crisis alimentaria global, parece sugerir que nos encontramos ante un acontecimiento sorprendente, inesperado, e imprevisible: algo repentino y muy localizado. Pero eso no es del todo cierto. La mala noticia del hambre es cualquier cosa menos nueva. Lo nuevo puede ser, en todo caso, la subida repentina de los precios de los alimentos que no hace sino agravar un problema que es endémico. Porque la situación de hambre de cientos de millones de nuestros congéneres es un fenómeno conocido, permanente, ampliamente divulgado y que no puede cogernos por sorpresa.

Por eso, antes de hablar de subidas de precios de los alimentos, hablemos del hambre. Y lo primero que constatamos es que el problema de la subnutrición se concentra tradicionalmente en un número limitado de países. Según el cuadro de la página siguiente, hay 18 países donde el porcentaje de personas subalimentadas está por encima del $35 \%$, de los cuales tan sólo tres no son africanos.

Evidentemente la desnutrición no afecta por igual a las poblaciones. Por eso también en países con cifras medias menos alarmantes hay amplios sectores de población gravemente afectados por el hambre, especialmente en aquéllos_en que la desigualdad es más notoria.

Para interpretar adecuadamente los datos estadísticos conviene distinguir entre hambre e inseguridad alimentaria. Hambrees, sencillamente, la situación de escasez de alimentos básicos. Inseguridad alimentaria es, como sugiere el término, la falta de seguridad en el acceso a los alimentos: en efecto, una persona puede no tener hambre en un momento determinado $y$, sin embargo, encontrarse en situación de inseguridad con respecto a la satisfacción de sus necesidades alimenticias, en cantidad y en calidad, en un futuro próximo. La inseguridad alimentaria es, por consiguiente, una situación de vulnerabilidad, un concepto vecino al de marginación.

\footnotetext{
${ }^{7}$ Porcentaje de población desnutrida sobre población total.
} 


\section{Cuadro I.- El hambre en el mundo. Países con más del $35 \%$ de población subnutrida ${ }^{8}$}

\begin{tabular}{|l|c|}
\hline AMÉRICA LATINA & $47 \%$ \\
\hline Haití & \\
CERCANO ORIENTE Y ÁFRICA DEL NORTE & \\
Yemen & $37 \%$ \\
\hline ÁFRICA SUBSAHARIANA & \\
Eritrea & $73 \%$ \\
Rep. Dem. del Congo & $72 \%$ \\
Burundi & $67 \%$ \\
Sierra Leona & $50 \%$ \\
Liberia & $49 \%$ \\
Zambia & $47 \%$ \\
Etiopía & $46 \%$ \\
Mozambique & $45 \%$ \\
República Centroafricana & $45 \%$ \\
Zimbabwe & $45 \%$ \\
República Unida de Tanzania & $44 \%$ \\
Angola & $38 \%$ \\
Madagascar & $38 \%$ \\
Rwanda & $36 \%$ \\
\hline ASIA & \\
República Democrática Popular de Corea & $35 \%$ \\
Tayikistán & $61 \%$ \\
\hline
\end{tabular}

Otro concepto vinculado a los anteriores es el de soberanía alimentaria. Se entiende por tal "el derecho de los pueblos a definir sus propias políticas y estrategias sostenibles de producción, distribución y consumo de alimentos que garanticen el derecho a la alimentación para toda la población, con base en la pequeña y mediana producción, respetando sus propias culturas y la diversidad de los modos

\footnotetext{
${ }^{8}$ Cf. Estrategia..., 69-70. En América Latina, contexto que nos resulta más familiar, por debajo de esa cifra crítica del $35 \%$, pero por encima del $20 \%$, se encuentran: Guatemala, Honduras, Nicaragua, Bolivia, Panamá y República Dominicana. Obsérvese la fuerte incidencia del problema en la región Centroamericana, que se agudiza particularmente en determinadas comarcas.
} 
campesinos, pesqueros e indígenas de producción agropecuaria, de comercialización y de gestión de los espacios rurales, en los cuales la mujer desempeña un papel fundamental" 9 .

Estos datos son más paradóiicos si se tiene en cuenta que la raíz última del problema del hambre no está en la falta de alimentos, sino en la falta de acceso a ellos por la pobreza de grandes segmentos de población. Esta afirmación no es gratuita: en la actualidad se genera un $17 \%$ más de calorías por persona que hace 30 años, a pesar de que la población se ha incrementado más del $70 \%$. Por lo tanto, el primer problema no es la disponibilidad de alimentos, sino la desigual distribución de los recursos necesarios para acceder al alimento. Es ahí donde radica verdaderamente el origen del hambre en nuestro mundo ${ }^{10}$.

Dada la relación entre pobreza y desnutrición, la subida actual de precios de los alimentos incrementará el número de pobres y el número de desnutridos en el mundo. Se calcula que mil millones de habitantes de nuestro mundo viven con un dólar diario o menos (pobreza absoluta). Como ese dólar se entiende no en su valor nominal sino según su capacidad adquisitiva, una aumento del precio de los alimentos básicos que, en una hipótesis moderada, podríamos cifrar en un $20 \%$, supondría que 100 millones más de personas se incorporarán a este nivel absoluto de pobreza. El poder de compra de "su dólar diario" habrá descendido considerablemente. Ello supone, para algunos países, retroceder una década en su lucha contra la pobreza.

¿Cuáles son, en síntesis, los efectos de la subida de los precios de los alimentos? Aparte del aumento obvio de la inflación, mencionemos dos efectos de la subida de precios en todo el mundo"1. El incremento de los precios afecta a todos, pero de una manera especial a las familias más pobres, dado que ellas destina la mayor parte de su ingreso a adquirir dichos alimentos básicos. Un segundo efecto se manifiesta en el gasto público: porque los gobiernos deben asignar ahora más recursos para asistir a la población más pobre y para apoyar la producción de esos alimentos que ellas precisan.

\footnotetext{
${ }^{9}$ Sobre todos estos conceptos, véase el excelente resumen de Estrategia..., 12-13.

${ }^{10}$ Cf. Estrategia..., 9.
}

11 Cfr. Unidad Regional de Asistencia Técnica (RUTA) (2008), Precios de los alimentos: Prospectiva. RUTA es una interagencia de cooperación especializada en cuestiones agrarias y rurales, con sede central en Costa Rica. España es socio de la misma. Cf. www.ruta.org (consulta del 29 de julio de 2008). 
El impacto del aumento del precio de los a limentos varía entre países y entre distintos grupos socioeconómicos ${ }^{12}$. Los países que son exportadores netos de alimentos deberían beneficiarse de la mejora en los términos de intercambio, aunque algunos de ellos están registrando pérdidas al prohibir las exportaciones para proteger a sus consumidores. Los países importadores netos, por su parte, tendrán que hacer esfuerzos para satisfacer su demanda interna de alimentos. Este último es el caso de casi todos los países de África: al ser importadores netos de alimentos, se verán seriamente afectados por el aumento de precios. Pero sabemos que todo esto está siempre condicionado por una variable ulterior: la de los intermediarios, que asumen un protagonismo importante en todas las relaciones comerciales.

Los crecientes y volátiles precios de los alimentos afectan a aquellos hogares con menor capacidad adquisitiva, es decir, a las personas pobres y expuestas a la inseguridad alimentaria. Los pocos hogares pobres que sean vendedores netos de alimentos resultarán beneficiados por los mayores precios, pero aquellos hogares que sean compradores netos de alimentos (es decir, la gran mayoría de las poblaciones pobres del mundo) se verán afectados. Deberá pasar mucho tiempo antes de que los ajustes en la economía rural, que pueden crear nuevas oportunidades de ingreso, lleguen a beneficiar a los pobres.

\section{Causas múltiples y complejas}

Una pregunta legítima que se hace mucha gente es: ¿̇quién tiene "la culpa" de lo que está pasando? ¡Ojalá hubiera "un" culpable! Pero ya hemos dicho que las cosas son, por desgracia, mucho más complejas. No hay una sola causa; más aún, la importancia relativa de las diferentes causas varía de un lugar a otro, por lo que no hay en absoluto claridad sobre cuál es la más determinante de las causas. Recordemos que estamos hablando de movimientos de precios en el mercado mundial: nada hay más volátil que los mercados, nada menos previsible.

Esta complejidad hace que los análisis sobre las causas difieran de unos autores a otros. Nosotros hemos optado por presentar un elenco lo más amplio posible, usando una clasificación que también algunos discutirán: factores relacionados con el incremento de la demanda; factores relacionados con la evolución de la oferta;

\footnotetext{
${ }^{12}$ Según una publicación del International Food Policy Research Institute (IFPRI): J. vON BRAUN (2008), "El aumento en los precios de los alimentos. ¿Qué hacer?", en: IFPRI (2008), Perspectiva de Políticas Alimentarias. Consultado en la página Web de RUTA, cf. supra.
} 
otros factores, relativos a políticas públicas o intereses de grupos, que tienen una incidencia relevante sobre el funcionamiento de los mercados. Aspiramos, más que a ofrecer una clasificación de las causas por orden de importancia, a suscitar en nuestros lectores la impresión de que estamos ante un fenómeno de gran complejidad. Subrayaremos también que las diferentes causas están relacionadas entre sí, por lo que no es fácil aislar cada una de ellas, salvo por la búsqueda de la simplicidad un tanto académica.

Para ambientar la descripción de causas que sigue, digamos desde ahora que estamos ante un caso "de libro" de aplicación de la ley de King, que se encuentra en todos los manuales de economía. Según ella, en los mercados agrarios, a oferta constante, pequeños incrementos de la demanda provocan aumentos mucho más que proporcionales en los precios; lo mismo ocurre cuando, manteniéndose constante la demanda, la oferta sufre bajadas, aun relativamente pequeñas. Es esto exactamente lo que está sucediendo en el mercado de los alimentos y se puede aplicar a otras materias primas como el petróleo.

\section{I. Causas que actúan por el lado de la demanda}

\section{a) El crecimiento demográfico}

El aumento de la población mundial ocasiona lógicamente una mayor demanda de alimentos. Dicho aumento demográfico no es un fenómeno reciente, ni nuevo; es más, precisamente en las últimas décadas se está produciendo un cierto descenso de los ritmos de dicho crecimiento, que conduce a pensar que nos acercamos a una cierta estabilidad demográfica en torno a los 10.000 millones de habitantes (8.000 millones en 2020). Este incremento exigiría aumentar la producción de alimentos entre un $40 \%$ y un $50 \%$. Ahora bien, las posibilidades de aumentar la superficie cultivable son relativamente reducidas en muchos lugares. Por otro lado, la creciente urbanización y las masivas migraciones del campo a la ciudad hacen que los precios de los alimentos sean más elevados: siempre fue más barato comer en el campo que en las ciudades...

¿Quién tiene, pues, "la culpa"? Como decía irónicamente Moisés Naïm refiriéndose más en general a la crisis económica mundial: "naturalmente, ¡como en todo últimamente!, los chinos". Y añade:

Y los indios, los indonesios y todos los pobres del mundo. Su culpa es que millones de ellos ahora tienen cómo comer más y mejor que antes, lo cual genera presiones inflacionarias. 
Esto está pasando, y ciertamente conlleva costos para todos. Pero es una tendencia que hay que aplaudir en vez de denigrar; estimular en vez de frenar. ${ }^{13}$

b) La estructura y los modelos del consumo de alimentos

No se trata, pues, sólo de que millones de personas coman más, sino de que comen distinto, comen mejor. Como dijo expresivamente José $M^{a}$ Sumpsi, subdirector de la FAO: "India y China quieren filete"14.

El rápido crecimiento económico en muchos países en desarrollo ha provocado un aumento en el poder de compra de los consumidores, lo cual se ha traducido en una mayor demanda de alimentos y, a la vez, en una recomposición de la demanda de productos básicos tradicionales hacia alimentos más costosos, como la carne y la leche. Este cambio en la dieta está provocando, entre otras cosas, un importante incremento en la demanda de granos utilizados para alimentar al ganado. Nos estamos, naturalmente, refiriendo al crecimiento de la demanda de los países emergentes. Los países desarrollados hemos asumido modelos de consumo de alimentos basados excesivamente en la carne; si los países emergentes adoptaran esa misma pauta, sería necesaria una superficie cultivable mundial ¡siete veces mayor a la actual!

\section{c) Los precios de la energía}

Los precios energéticos conocen alzas espectaculares derivadas también de los grandes incrementos de demanda. Los datos históricos demuestran que dichos precios están cada vez más correlacionados con los precios de los productos agrícolas. Por eso, entre otras razones, también las bajadas del crudo en agosto y septiembre han contribuido a suavizar las tensiones alcistas de dichos precios. Al mismo tiempo, el alto precio de los productos energéticos ha contribuido a encarecer la producción agrícola al aumentar el costo del cultivo mecánico, de insumos como los fertilizantes e insecticidas y del transporte de insumos y productos. Este último factor es, al mismo tiempo, un factor relativo a la oferta, ya que afecta a la manera cómo se producen los alimentos.

${ }^{13}$ M. NAïM (2008), "¿Quién hundió la economía mundial??", El País, 27 de julio.

${ }^{14}$ El País, 21 de abril de 2008. 


\section{d) Los biocombustibles}

Estrechamente relacionado con la anterior subida de precios del petróleo está el hecho de que el gobierno de Estados Unidos está otorgando subsidios a los agricultores para que dediquen sus cosechas a la producción de energéticos; y los agricultores de este país están destinando masivamente sus cultivos, en particular el del maíz, a la producción de biocombustibles, a expensas de los cultivos de soja y trigo. Casi el 30 por ciento de la cosecha de maíz de Estados Unidos será destinada a la producción de etanol en 2008, en lugar de enviarse a los mercados mundiales de alimentos para consumo humano y animal. Según Malagoli, del aumento de 40 millones de toneladas de maíz en 2008 la producción de etanol consumirá nada menos que 30 millones, principalmente en Estados Unidos. Como veremos esta es una de las medidas más polémicas, sobre la que se ejercen numerosas presiones.

En palabras del ya citado Moisés Naïm:

Además, no es cierto que la actual ola de inflación mundial es causada principalmente por los nuevos consumidores. Las causas de la inflación tienen más que ver con las políticas de los países ricos que con los hábitos de los consumidores pobres. Las estadísticas muestran que los subsidios al etanol por ejemplo, encarecen más la comida que el aumento en el consumo de alimentos en los países pobres ${ }^{15}$.

\subsection{Por el lado de la oferta}

\section{a) Cambio climático y malas cosechas}

Otro factor que ha contribuido al alza del precio de los alimentos son las malas cosechas derivadas del mal clima y de los cambios climáticos que están en el origen de las "crisis hídricas". Todo el mundo cita la grave sequía que sufrió Australia, uno de los más grandes productores de trigo, y que ha afectado a su vez a la producción de este grano en el mundo. También se mencionan las malas cosechas de Ucrania (otro gran productor) o de Nueva Zelanda.

${ }^{15}$ M. NAÏM, art. c. 
b) La competencia por el uso del suelo

Asistimos a una reducción del suelo agrícola, que es consecuencia del aumento de la demanda del suelo para otros fines. Téngase en cuenta que la tierra cultivable es un bien inelástico donde los haya..., pero hoy una buena proporción del mismo se destina a usos no alimenticios: a infraestructuras, a urbanizaciones, a aeropuertos, a centros comerciales, etc. Por otra parte, una proporción creciente de tierras de los países menos desarrollados se destina a cultivos orientados al consumo de los habitantes de los países ricos: es el caso, por ejemplo, de la producción de flores en Kenya para el mercado europeo o de la transformación de los campos de arroz de la India en cultivos de camarones. Es la población local quien sufre las consecuencias de la consiguiente disminución en la oferta de alimentos.

\subsection{Intereses políticos presentes en los mercados}

Pero los mercados no funcionan normalmente según los modelos teóricos. En concreto, es importante en ellos la intervención de los gobiernos y la acción de las grandes empresas o grupos empresariales. A todas las actuaciones de este tipo queremos referirnos ahora, porque sin tenerlas en cuenta sería casi imposible entender lo que ocurre en los mercados alimentarios. Al exponerlas las hemos ordenado, no según un criterio de importancia, sino por orden cronológico: creemos que así se comprende mejor cómo han ido incidiendo en esta compleja problemática que estamos analizando.

a) La supresión de las políticas agrícolas como consecuencia de los ajustes estructurales

Nos referimos ahora a una cuestión de más amplio alcance cual es la puesta en marcha de los programas de ajuste estructural y de reforma institucional, que buscaban -allá por los años 90- mejorar la eficiencia del sector público. Entre sus consecuencias debe contarse el notorio debilitamiento de las políticas agrarias, que se justificó desde una cierta confianza en que el sector privado se encargaría de la prestación de servicios tradicionalmente encomendados a la administración del Estado. Los resultados no respondieron a estas expectativas.

Sabemos que estos programas, propiciados por el Fondo Monetario Internacional y por el Banco Mundial, fueron especialmente duros con los países en vías de desarrollo, y particularmente con los más endeudados; y que enfatizaban hasta el paroxismo entre otras cosas la necesidad de las políticas-macro de disminu- 
ción del gasto público hasta propugnar en la práctica ly, en algunos casos, en la teoría) el abandono de políticas sectoriales tales como las políticas agrarias y de desarrollo rural.

Es difícil no admitir que esa falta de atención por parte del sector público al mundo rural y a sus necesidades durante más de una década no esté detrás de los problemas alimenticios que sufren los países menos desarrollados. La relación causa/efecto entre ambos fenómenos puede intuirse desde una elemental teoría del mercado y de las dificultades de éste para responder a las necesidades reales cuando los agentes económicos son muy desiguales en poder y en capacidad adquisitiva.

\section{b) Las trabas a las exportaciones}

Algunos países como Argentina, Bolivia, Camboya, China, Egipto, Etiopía, India, Indonesia, Kazajstán, México, Marruecos, Rusia, Tailandia, Ucrania, Venezuela y Vietnam han querido mantener la oferta interna de alimentos restringiendo las exportaciones de éstos o limitando sus precios, o bien con una combinación de ambas medidas. Imponiendo trabas arancelarias y cupos a la exportación de alimentos han pretendido luchar contra la amenaza de escasez: pero eso está contribuyendo a la subida de los precios en los mercados internacionales ${ }^{16}$. Por ejemplo, en China se han prohibido las exportaciones de arroz y maíz; en la India, las de leche en polvo; en Bolivia, las de aceite de soja a Chile, Colombia, Cuba, Ecuador, Perú y Venezuela; en Etiopía está prohibida la exportación de los principales cereales. Los países que siguen ese tipo de políticas, al intentar proteger el mercado interno dificultando las exportaciones, provocan un descenso de la oferta mundial, con el consiguiente efecto de aumento más que proporcional (Ley de King) de los precios mundiales.

En otros países se están reduciendo las restricciones a las importaciones: en Marruecos, por ejemplo, se han reducido los aranceles a la importación de trigo, de 130 por ciento al 2,5 por ciento; en Nigeria se hizo lo mismo.

\footnotetext{
${ }^{16}$ No se ha destacado quizá suficientemente en los medios de comunicación el hecho de que la grave crisis política que ha vivido Argentina tiene su origen en la oposición de los agricultores a la imposición de aranceles a la exportación de granos, lo que les impedía aprovechar las ventajas de las subidas internacionales de precios. Suponemos que la política del gobierno de la señora Kirchner pretendía salvaguardar la seguridad alimentaria de los consumidores de su propio país... impidiendo a los productores las ganancias derivadas de las subidas de precios en los mercados mundiales. En unos enfrentamientos sin precedentes, los poderosos y articulados intereses agrarios argentinos se movilizaron hasta límites insospechados para luchar contra esas medidas, logrando provocar su revocación.
} 
En realidad estas medidas sobre exportaciones e importaciones podrían incluirse, no entre las causas de la crisis, sino entre sus consecuencias ya que son medidas orientadas a minimizar sus efectos. Pero lo que hacen es agravarla.

\section{c) Monopolios y especulación}

La grave crisis financiera resultante de la ruptura de la burbuja de las hipotecas de alto riesgo o hipotecas basura ${ }^{17}$ hizo que muchos inversores buscaran seguridad en otros mercados que presentaran mejores perspectivas. El de los alimentos fue uno de ellos. De este modo hemos visto cómo la especulación, un fenómeno tan frecuente en el actual contexto de globalización y de movimientos fulgurantes de capitales internacionales sumamente sensibles al riesgo, ha venido a alimentar las tensiones alcistas de los precios de los alimentos. Es más, el notable desarrollo de productos financieros derivados, que caracteriza estas últimas décadas, se está intensificando ahora con las operaciones relativas a las producciones agrarias, lo que aumenta el atractivo de los especuladores.

Por otra parte, las grandes multinacionales de la alimentación han presionado mucho sobre el mercado, acaparando existencias con el fin de garantizar sus "stocks" de materia prima para la producción bienes elaborados. Para ello han recurrido con profusión a los contratos a término o de futuro que, naturalmente, también influyen sobre el mercado de contado, en mercados donde además la tendencia al monopolio o al oligopolio es muy fuerte.

\section{d) Nuevas políticas agrícolas y descenso de "stocks"}

La evolución de las políticas agrícolas, sobre todo la de los países más influyentes ha influido también en esta situación. Naturalmente aquí es inevitable una referencia a la Política Agrícola Común de la Unión Europea.

Desde la llamada Reforma Mc Sharry de 2000, y más aún desde la reforma más radical de 2003, se ha pasado de una política incentivadora de la producción a una política que tiende a frenar la producción, el gasto y los excedentes, así

\footnotetext{
17 Véase sobre este tema nuestro comentario editorial: CONSEJO DE REDACCIÓN (2007), "Claves para comprender la crisis financiera internacional". Revista de Fomento Social, $\mathrm{n}^{\circ} 248$, octubre-diciembre, 515-535. Ya nos referíamos allí a la subida de los precios de las materias primas: cf. pp. 521 y 525. El punto álgido de esta crisis parece localizarse en octubre de 2008, con tremendos impactos en las bolsas y actuaciones masivas de los gobiernos para salvar los sistemas financieros.
} 
como a disminuir los niveles de precios protegidos a los agricultores. Más aún, ya no se paga en función de la cantidad producida (para evitar que la subvención produzca excedentes tan caros como innecesarios): se opta, en cambio, por garantizar una ayuda directa a los agricultores que es independiente de lo que cada uno produce. De este modo se aseguraba una renta sin incentivar la producción, al tiempo que se disminuían drásticamente los "stocks", cuyo mantenimiento era ruinoso. Se acabaron también las exportaciones subvencionadas (las famosas y tan denostadas "restituciones" de la PAC), que tendían a hacer bajar los precios en los mercados mundiales al inundarlos con productos excedentarios vendidos por debajo del coste de producción (exportaciones "dumping").

Esta política está dando los frutos deseados en la Unión Europea. Pero, paradójicamente, está contribuyendo a agravar el problema de los precios agrícolas al carecerse ya de esas fuertes reservas de seguridad de los alimentos principales y las consiguientes exportaciones a bajo precio, que servían como factor de equilibrio en los mercados mundiales. Nos atrevemos a pensar que, si la Unión Europea hubiera dispuesto de los "stocks" que se llegaron a acumular en los años 1980, se hubiera reducido significativamente el alcance de la crisis.

\section{e) Los subsidios y el fracaso de la Ronda Doha}

El 29 de julio Pascal Lamy, director general de la Organización Mundial del Comercio, anunciaba el fracaso de las negociaciones comerciales multilaterales de la Ronda Doha, que habían reunido en Ginebra durante nueve días a delegaciones de 35 países. Cuando parecía que el acuerdo estaba al alcance de la mano, fue precisamente la cuestión agrícola la que llevó al traste un paquete de 20 medidas de las que 18 ya estaban consensuadas: fueron los Estados Unidos los que rechazaron tajantemente como proteccionista la propuesta de China e India de que los países pobres y fuertemente poblados pudieran establecer mecanismos de protección para productos agrícolas con objeto de defenderse de la invasión de importaciones baratas provenientes de países industrializados.

Es difícil pronosticar todavía los efectos de este grave fracaso, que se produce precisamente cuando parecía que iba a alcanzarse un acuerdo histórico sobre la disminución de las ayudas y subsidios a los productores europeos y norteamericanos, tan solicitada por los países en desarrollo y por multitud de organizaciones solidarias internacionales.

¿Quién se opone a esos subsidios? Naturalmente, no los consumidores de los países pobres, que dispondrían de una mayor oferta de productos a precios subsidiados. 
Se oponen los exportadores de los países productores de bienes agrícolas, que ven en esos subsidios un factor de competencia que les perjudica. Pero estos subsidios han sido considerados por muchos además como un ataque a la soberanía alimentaria de los países pobres, lo que es especialmente cierto en cuanto a las restituciones o subvenciones a las exportaciones de excedentes europeos (que en ocasiones han provocado el hundimiento de precios en los mercados locales) y que han desaparecido casi por completo, en el caso europeo.

El tema de las ayudas es diferente. Resulta mucho más difícil demostrar -a pesar de muchas afirmaciones excesivamente contundentes ${ }^{18}$ - la influencia automática de las nuevas modalidades de ayudas directas sobre el aumento del hambre y de la inseguridad alimentaria. En todo caso, su disminución sensible a medio plazo estaba prevista en el paquete de las citadas frustradas negociaciones de Ginebra.

\section{Una reflexión ética}

Antes de preguntarnos qué se puede hacer ante la situación analizada es conveniente detenerse en algunos criterios éticos en que fundamentar esta actuación y desde los que orientarla. Podría parecer superfluo, argumentando que basta una mínima sensibilidad humana para reaccionar ante los hechos descritos. Pero sabemos que la voluntad, por muy buena que sea, no basta: hacen falta mecanismos concretos, hace falta voluntad política, hace falta la colaboración de todos. Precisamente porque las causas son complejas, las respuestas no pueden ser simples.

Y lo primero que hay que dejar claro es que la seguridad alimentaria tiene que ver con los derechos humanos. Ya la Declaración Universal de 1948 reconoce el derecho a una alimentación adecuada como complemento central del derecho a un nivel de vida digno:

Toda persona tiene derecho a un nivel de vida adecuado que le asegure, así como a su familia, la salud, el bienestar, y en especial la alimentación, el vestido, la vivienda, la asistencia médica y los servicios sociales necesarios (art. 25, 1).

Es importante recordar que estamos ante un derecho social. Éstos no sólo han de ser reconocidos por el Estado, sino que éste ha de poner los medios para que los ciudadanos puedan hacerlos realidad. Por esta razón el Pacto Internacional de los

\footnotetext{
${ }^{18}$ Como la de nuestros buenos amigos de Intermón OXfaM. Véase por ejemplo: G. Fanjul (2008) "Lecciones de una crisis", El País, 5 de mayo.
} 
Derechos Sociales, Económicos y Culturales, aprobado en 1966, incluye el derecho a la alimentación, concretando incluso las obligaciones de los poderes públicos:

Los Estados Partes en el presente Pacto, reconociendo el derecho fundamental de toda persona a estar protegida contra el hambre, adoptarán, individualmente y mediante la cooperación internacional, las medidas, incluidos programas concretos, que se necesitan para:

a) Mejorar los métodos de producción, conservación y distribución de alimentos mediante la plena utilización de los conocimientos técnicos y científicos, la divulgación de principios sobre nutrición y el perfeccionamiento o la reforma de los regímenes agrarios de modo que se logre la explotación y la utilización más eficaces de las riquezas naturales;

b) Asegurar una distribución equitativa de los alimentos mundiales en relación con las necesidades, teniendo en cuenta los problemas que se plantean tanto a los países que importan productos alimenticios como a los que los exportan (art. 11, 2).

Del texto cabe destacar al menos estos dos detalles: la actuación del Estado se concreta, no sólo en la producción, sino también en la distribución de alimentos; la tarea, que es competencia en primer lugar de cada Estado dentro de su territorio (como corresponde a los derechos sociales) ha de hacerse en el marco de la cooperación internacional. Ambos extremos adquieren una relevancia especial en las condiciones actuales.

Mucho más recientemente, ya en 1999, nos encontramos con otro texto de la Comisión Económica y Social de la ONU. Se trata de un "Comentario" ("General Comments") al art. 11 del Pacto que acabamos de citar sobre "El derecho a una alimentación adecuada" (12 mayo 1999). Lo interesante del mismo es cómo concreta los deberes de los Estados en tres: the obligations to respect, to protect and to fulfil (las obligaciones de respetar, de proteger y de realizar). El contenido de cada una de ellas se explica como sigue:

- Respetar: no adoptar medidas políticas o de otro tipo que puedan reducir de modo significativo la disponibilidad de alimentos o que impida el acceso a la alimentación adecuada a las poblaciones vulnerables.

- Proteger: incluye la obligación del Estado de asegurar que las actuaciones de otros gobiernos, entidades privadas o individuos, incluidas las empresas multinacionales, no amenacen la disponibilidad de alimentos o priven a las personas del acceso a la alimentación adecuada. 
- Realizar: en este punto se distinguen dos niveles diferentes:

- Facilitar: que el Estado emprenda de forma proactiva iniciativas para reforzar en las personas el acceso a los recursos que les permiten vivir, incluida la seguridad alimentaria. Se trata en el fondo de ayudar a la capacitación de las personas a largo plazo para que éstas puedan alimentarse por sí mismas.

- Proporcionar: que el Estado facilite directamente los medios de alimentación a aquellas personas o grupos que, por razones que escapan a su control, son incapaces de procurárselos por sí mismos; se incluyen aquí las víctimas de los desastres naturales o de otros desastres.

Esta enumeración describe muy bien lo que es la responsabilidad de los poderes públicos en relación con los derechos sociales: el contenido central es, sin duda, capacitar a las personas para que puedan valerse por sí mismas también en esta tarea de la alimentación (otra forma de "empoderar"). Pero hay otros aspectos a tener en cuenta: desde los casos de necesidades urgentes en los que no hay tiempo material para "empoderar" hasta los obstáculos que no dependen del Estado mismo, sino de agentes externos, y que éste tiene obligación de afrontar (una forma más de "defensa").

Hasta aquí, lo que corresponde a un Estado como consecuencia de sus funciones en relación con la población sobre la que ejerce la soberanía. Sabemos que ése es el marco en que se formuló la Declaración de 1948 y los dos Pactos de 1966 que la desarrollaron y aplicaron. Pero esto no basta. La globalización de los mercados reduce la capacidad de los Estados para ejercer todas sus responsabilidades en relación con los derechos sociales. Por eso se impone, también como una exigencia de carácter moral, la colaboración de todos los Estados para afrontar problemas que son de todos. Desde que la globalización se hizo un fenómeno incuestionable e imparable, la solidaridad empezó a ser algo más que un valor moral o una actitud personal: se convirtió en un verdadero criterio de organización social e internacional. La presentación que de la solidaridad hizo Juan Pablo Il en su carta encíclica sobre el desarrollo, como la respuesta mejor a la creciente interdependencia entre los pueblos (un término que preanuncia ya el de globalización), sigue siendo de gran actualidad:

Ante todo se trata de la interdependencia percibida como sistema determinante de relaciones en el mundo actual, en sus aspectos económico, cultural, político y religioso, y asumida como categoría moral. Cuando la interdependencia es reconocida así, su correspondiente respuesta, como actitud moral y social, y como "virtud", es la solidaridad. 
Ésta no es, pues, un sentimiento superficial por los males de tantas personas, cercanas o lejanas. Al contrario, es la determinación firme y perseverante de empeñarse por el bien común; es decir, por el bien de todos y cada uno, para que todos seamos verdaderamente responsables de todos ${ }^{19}$.

Esta expresión final (para que todos seamos verdaderamente responsables de todos) da un contenido muy concreto a un término que, de tanto usarse, corre el peligro de cargarse de ambigüedad. La solidaridad es lo que está detrás del esfuerzo de colaboración de los gobiernos cuando se reúnen en las grandes conferencias internacionales que tanto han proliferado en los últimos tiempos. Es cierto que se mezclan otros móviles o intereses: unos, basados en los egoísmos particulares; otros, vinculados con las amenazas que se ciernen sobre la humanidad toda. Pero la solidaridad es una motivación más, de claro carácter ético, para hacer frente a estos grandes retos, como es el que nos ocupa en estas páginas.

De hecho, son muchas las iniciativas puestas en marcha para hacer frente a la crisis alimentaria. La FAO ha desempeñado un papel, que podría ser más significativo si se viese más apoyada por la voluntad política de los gobiernos como cauce eficaz para la cooperación. En seguida haremos alusión a alguna de estas iniciativas. De momento sólo queremos dejar constancia de esta segunda línea de responsabilidad que, más allá de la del Estado dentro de su territorio, afecta a todos los Estados en relación con la humanidad toda.

Ahora bien, la solidaridad no es tarea sólo de los poderes públicos. Obliga también a los ciudadanos del mundo. En una humanidad más sensible a estos problemas de alcance planetario también se multiplican las iniciativas. Las más específicas son las que proceden del tercer sector: organizaciones que buscan no los propios intereses particulares de sus miembros, sino los de colectivos más vulnerables o los intereses generales de la humanidad.

Tampoco podemos excluir de esta responsabilidad a las organizaciones lucrativas: es cierto que su objetivo primario son los intereses legítimos de sus componentes, pero eso no pueden hacerlo ignorando los efectos de su acción sobre otros colectivos humanos. La responsabilidad social empresarial apunta a esto: no se pretende convertir una empresa en una entidad de beneficencia, pero sí de que asuma que el gran poder que dichas instituciones tienen en el mercado mundial es fuente de una correlativa responsabilidad sobre los efectos, pretendidos o no, de su acción.

19 JuAN PABlo II (1987), carta encíclica Sollicitudo rei socialis, n. 38. 
Desgraciadamente lo que contemplamos, sobre todo en los mercados mundiales, es una praxis bastante despiadada de las grandes empresas, que buscan sus propios beneficios sin reparar en medios. Ya quedó denunciado cuando analizamos las causas de los fuertes incrementos de los precios alimenticios. Y esto es una razón más para urgir a los que cuidan de los intereses públicos: porque están obligados a intervenir en los dos niveles indicados -el Estado dentro de su territorio y los Estados todos a través de la cooperación-, al menos para evitar que la actuación de muchas empresas multinacionales cause perjuicios directos a la seguridad alimentaria de los pueblos y colectivos más pobres del planeta.

\section{5. ¿Qué se está haciendo? ¿Qué se puede hacer?}

El diagnóstico que hicimos más arriba permite prever que las soluciones no son fáciles ni pueden reducirse a unas pocas acciones o a contados actores. Esta convicción es la que preside las propuestas y reflexiones que siguen. Hemos preferido esta presentación más amplia porque creemos responde mejor a la complejidad del fenómeno y a la multiplicidad de sus causas.

\section{I. Un fenómeno que no es nuevo: los Objetivos del Milenio}

Efectivamente, no nos encontramos ante un fenómeno nuevo. Desde hace años, la tan grave cuestión de la desnutrición está presente en la agenda del desarrollo. Por referirnos solamente a un archiconocido plan mundial, en septiembre de 2000, en la Cumbre del Milenio de las Naciones Unidas, los dirigentes mundiales acordaron una serie de medidas para combatir la pobreza, el hambre, las enfermedades, el analfabetismo, la degradación del medio ambiente y la discriminación contra la mujer y crear una asociación mundial para el desarrollo.

Este pacto mundial, que se conoce como los Objetivos de Desarrollo del Milenio (ODM), fue acordado por todos los países miembros de las Naciones Unidas: en este sentido, es una buena expresión de esa solidaridad mundial a la que aludíamos arriba. En la Declaración de la Cumbre del Milenio se esbozó además un plan, igualmente acordado por consenso, en cuanto a la forma de aplicar los acuerdos: en ella se expresaba el compromiso de prestar más atención a los derechos humanos, a la buena gestión de los asuntos públicos y a la democracia, así como a la prevención de los conflictos y a la consolidación de la paz. A partir de entonces todas las agendas del desarrollo incluyen los objetivos del milenio como una referencia obligada en sus planes de acción de lucha contra la pobreza. 
Sea cual sea el grado de avance en la consecución de los objetivos acordados, lo primero que hay que destacar es que nos encontramos ante objetivos "de mínimos", una especie de "rebaja" (quizás inevitable en aras del consenso) de lo que deberían ser objetivos de un verdadero desarrollo humano concebido como "ampliación de capacidades" (Amartya Sen). Aunque haya que luchar por alcanzarlos, el desarrollo humano no puede conformarse con semejantes metas.

En todo caso, es destacable en los ODM el hecho de establecer, no sólo objetivos, sino metas medibles y plazos para su cumplimiento. Así, por ejemplo, el primero de dichos objetivos, que es el que a nosotros nos interesa aquí, se formula así: Erradicar la extrema pobreza y el hambre. Y se concreta en estas dos metas:

a) Reducir a la mitad, entre 1990 y 2015, el porcentaje de personas cuyos ingresos sean inferiores a US\$ 1 por día. Para calibrar lo que está en juego, recuérdese que en 1999, año de referencia, 1.241 millones de personas se encontraban en esta situación.

b) Reducir a la mitad, entre 1990 y 2015, el porcentaje de personas que padecen hambre. También como punto de referencia valga recordar que el hambre y la malnutrición afectaban hacia el año 2000 a más de 800 millones de habitantes del planeta.

Los datos disponibles sobre el grado de cumplimiento de los ODM muestran que los resultados son dispares. Además existe una estrecha relación entre ellos: concretamente la lucha contra el hambre tiene un carácter estratégico, ya que, sin avanzar en él es difícil progresar en otros. Por otra parte, el avance está siendo desigual según grandes áreas, y desde luego el itinerario seguido por África subsahariana no da pie para grandes esperanzas. Una prueba de ello es el estallido de la crisis alimentaria en estos años.

\subsection{Primeras medidas de urgencia}

Ya mencionamos al comienzo de estas páginas la cumbre mundial, organizada por la FAO en junio pasado sobre el tema "Seguridad alimentaria y mundial: los desafíos del cambio climático y de la bioenergía". Entre los temas incluidos en la agenda ocupaba el primer lugar el aumento de los precios mundiales de los productos agrícolas ${ }^{20}$. Aunque los resultados de la cumbre, incluida su declaración

${ }^{20}$ Los restantes temas de la cumbre eran: la posibilidad de recurrir a los organismos genéticamente 
final, han sido recibidos en general con cierto escepticismo, la reunión misma ha tenido cierto efecto de sensibilización, que se ha traducido en algunas acciones concretas.

Por ejemplo, la Unión Europea reaccionó rápidamente. En efecto, la Comisión Europea ha respaldado el 18 de julio un plan para enviar a los agricultores de África mil millones de euros de los subsidios agrarios que la Unión Europea no ha utilizado. El objetivo de la partida, aprobada con la oposición de varios Estados miembros de la Unión, es ayudar a que los agricultores africanos afronten el alza de los precios y la crisis alimentaria.

En la misma cumbre de Roma, el presidente del Gobierno español ya se había comprometido a que nuestro país destinará 500 millones de euros hasta 2012 para mejorar la productividad de los pequeños agricultores de los países pobres y ayudarles a que puedan producir los alimentos que necesita la humanidad ${ }^{21}$.

Por su parte, los países directamente afectados han puesto en marcha algunas medidas de urgencia. Entre ellas: la reactivación o aumento en la cuantía de la ayuda alimentaria a la población más pobre y el apoyo la producción de alimentos básicos. Este apoyo se instrumenta por distintos cauces: se está subsidiando la entrega de semillas y fertilizantes, se está suministrando asistencia técnica, se están flexibilizando las normas para la concesión de crédito, se prometen seguros para las cosechas con primas subsidiadas por el Estado. Son reacciones que se repiten con variantes en distintos países y áreas afectadas.

modificados (OGM), el impacto de la utilización de los productos agrícolas para la producción de energía y carburantes (biocombustibles), las consecuencias del cambio climático sobre el sector agrícola mundial.

${ }^{21}$ Este género de promesas generosas honran a nuestro país; la cuestión está en saber si la cooperación española en general, de la Agencia Española de Cooperación Internacional para el Desarrollo (AECID) en particular, tienen capacidad de respuesta y rapidez de reflejos para gastar bien; este tipo de declaraciones tiene el peligro de desencadenar procesos de gastos rápidos no necesariamente eficientes. Son muy atinadas al respecto las reflexiones de uno de nuestros mayores expertos en la materia, José Antonio Alonso (2008), "España en guerra contra el hambre", El País, 5 de julio. Conocemos de cerca con cuánta prisa se desencadenaron acciones "para acelerar el gasto" de la cooperación española en algunos países centroamericanos especialmente afectados por el problema que nos ocupa. 


\subsection{Países pobres y países ricos: tareas pendientes}

Como las responsabilidades son de unos y otros, de los pueblos en desarrollo y de los ya desarrollados, nos ha parecido que sería ilustrativo contraponer lo que corresponde a unos y a otros.

Los gobiernos de los países pobres tienen que actuar en primer lugar con acciones a corto plazo. Se trata de programas de protección social orientados a la seguridad alimentaria de los grupos de población más pobres, tanto en áreas urbanas como rurales. Algunos de estos grupos más pobres no tienen fácil acceso a los mercados y, por ello, ni siquiera sienten los efectos del aumento en el precio de los alimentos: necesitan algo tan elemental como las transferencias de alimentos o planes de nutrición para infantes. Pero hay millones de consumidores pobres de áreas urbanas y rurales que son compradores netos de alimentos y cuya exposición al mercado es mayor: en estos casos los altos precios internacionales pueden afectar gravemente su subsistencia. Evidentemente estos grupos precisan de una ayuda directa y urgente. Entre ellas es posible que haya que optar por algunas medidas más rudimentarias, como la prohibición a las exportaciones y los subsidios a las importaciones.

Pero no basta con acciones a corto plazo. En las últimas décadas las inversiones en áreas rurales han estado bastante olvidadas. Mientras tanto, los agricultores de muchos países en desarrollo disponen de una infraestructura inadecuada en cuanto a carreteras, electricidad, comunicaciones, suelos pobres, falta de plantas de almacenamiento y procesamiento, y acceso escaso o nulo a tecnologías agrícolas que les permitan aumentar sus ganancias y mejorar sus niveles de subsistencia. La crisis actual deja en evidencia estas deficiencias que impiden un crecimiento agrícola a largo plazo. Esta es la mejor estrategia a adoptar por los gobiernos de los países en desarrollo: supone aumentar sus inversiones en investigación y extensión agrícola, en infraestructura rural y en accesibilidad a los mercados para los pequeños agricultores. Esta línea de actuación tropezará en muchos países con los intereses de los consumidores urbanos, que se han manifestado recientemente en fuertes disturbios ocasionados por el alza de los precios de los alimentos en varios países.

Tales iniciativas a largo plazo tienen como último objetivo lograr la soberanía alimentaria. Y ello implica, no sólo disponer de alimentos y con precios asequibles, sino garantizar el acceso a ellos de los grupos con menos recursos. En este sentido la lucha contra la pobreza redundará en más capacidad para hacer frente a las necesidades de alimentos. 
Si de los países pobres pasamos ahora a los países ricos, una primera responsabilidad de éstos es contribuir a las políticas que acabamos de exponer para los primeros. En este sentido, los donantes del Norte deberán ampliar sus planes de ayuda al desarrollo e incluir programas de protección social, nutrición infantil y ayuda alimentaria, en donde sean necesarios. Y deberán también incrementar las inversiones orientadas a la agricultura, los servicios rurales, la ciencia y la tecnología. Pero ahí no acaban sus responsabilidades: otras, las más importantes quizás, se refieren a los efectos de sus políticas sobre la viabilidad de la soberanía alimentaria de los países pobres.

Quizás el punto más delicado y más decisivo es la eliminación por parte de los países desarrollados de las barreras comerciales a la agricultura. Es cierto que se ha avanzado en la reducción de los subsidios a la agricultura y en otras políticas que afectan al comercio en los países en desarrollo. Pero quedan todavía muchos subsidios y políticas con los que no pueden competir los países pobres. El último fracaso de la Ronda Doha en Ginebra pone de manifiesto que, aunque el acuerdo parece estar cerca, no acaba de consumarse. Pero las oportunidades pueden ser mejores ahora: el riesgo políitico para las autoridades de los países desarrollados es menor, mientras que la urgencia de la crisis puede servir de estímulo para desbloquear las reticencias de siempre.

Una cuestión más reciente, pero que hoy adquiere una actualidad indiscutible es la relativa a la producción de biocombustibles. Este tema hay que considerarlo en todas sus dimensiones: porque es cierto que estos productos son un alivio como alternativa al petróleo, pero su producción tiene repercusiones indiscutibles y problemáticas sobre los precios de los alimentos. En palabras de Amartya Sen: "los estómagos de los que pasan hambre han de competir además con los depósitos de combustible" ${ }^{\prime 22}$. Concretamente los subsidios a los biocombustibles en Estados Unidos, y al etanol y a los biocombustibles en Europa, han afectado a los mercados mundiales de alimentos: funcionan como un impuesto implícito a los alimentos básicos, de los que más dependen los sectores pobres de la población. Por eso los países desarrollados deben eliminar estos subsidios a la producción de biocombustibles y abrir sus mercados a los países en condiciones de exportarlos, como es el caso de Brasil.

${ }^{22}$ A. SEN (2008), "The Rich Get Hungrier", The New York Times, 28 de mayo. 


\subsection{Vuelta a las políticas agrícolas}

Este punto merece una atención especial, no sólo por su relación directa con el tema que nos ocupa, sino además por la actualidad que ha adquirido a raíz de la publicación del Informe del Banco Mundial de 200723. Por primera vez en los últimos 25 años el Banco Mundial se pronuncia en favor de la reinstauración de políticas agrícolas. Citamos textualmente:

Los gobiernos y los donantes reconocen cada vez más ampliamente que la agricultura debe ser una parte fundamental del programa del desarrollo, ya sea para generar crecimiento en los países agrícolas o para reducir la pobreza rural y abordar los problemas ambientales en todo el mundo. La mayor cantidad de oportunidades y la mayor disposición a invertir en agricultura que se observan en la actualidad justifican la visión optimista de que los programas de agricultura para el desarrollo pueden llevarse adelante. No debe desperdiciarse la oportunidad que se presenta, puesto que el éxito brindará enormes beneficios para la consecución de los objetivos de desarrollo del milenio y aún más allá (p. 27).

Este mensaje no tendría tanto "morbo", si se nos permite la expresión, si no fuera porque la institución que así lo proclama, durante varios lustros ha defendido en la teoría y en la práctica la aplicación de duros programas de ajuste estructural en los países en vías de desarrollo, particularmente en los países más endeudados: y esto ha supuesto, entre otras cosas, la eliminación progresiva de las políticas sociales y agrícolas. Este informe constituye, por tanto, un "mea culpa" -eso sí, implícito y velado- y retractarse de lo que los autores llaman "el sesgo antiagrícola de las políticas macroeconómicas", que se ha reducido "gracias a la introducción de reformas económicas más amplias", que tanto daño han hecho en muchos países.

El Informe comienza con esta afirmación programática: Es hora de volver a colocar este sector en el centro del programa del desarrollo (p. 1).

Las políticas agrícolas implican la intervención del Estado y, consecuentemente, la pérdida de confianza en el mercado para garantizar un crecimiento económico con equidad: El mercado presenta deficiencias generalizadas, en especial en los países agrícolas, y se requieren políticas públicas para garantizar la obtención de los resultados sociales deseados. El Estado desempeña un papel importante en el desarrollo del mercado (p. 25). De crecimiento con equidad no se habla,

${ }^{23}$ BANCO MUNDIAL (2007), Informe sobre el desarrollo mundial 2008. Agricultura para el desarrollo, Washington, Banco Mundial. Véase recensión detallada del mismo en nuestra revista de J. J. ROMERO RodríGuez (2007), Revista de Fomento Social, n² 248, octubre-diciembre, 641-648. 
pero hay textos que presuponen ese objetivo: ... se requiere no sólo invertir en las zonas menos favorecidas para combatir la pobreza extrema, sino también dirigir los esfuerzos a la gran cantidad de pobres que habitan en las zonas favorecidas (p. 12). Para ello se reconoce al Estado un protagonismo que durante décadas se le negó: ... resulta imperioso fortalecer la capacidad del Estado en sus nuevas funciones de coordinador de sectores y de socio del sector privado y la sociedad civil. En la mayor parte de los países, hace falta emprender reformas de gran magnitud en los ministerios de agricultura con el objeto de redefinir sus funciones y desarrollar nuevas capacidades (p. 25).

Esta selección de citas permite concluir $-y$ nos congratulamos de que el Banco Mundial haya llegado a tal conclusión- que lo que hace falta no es menos política agrícola sino más. Pero políticas adaptadas a las realidades de cada país o región. Estamos ante un poderoso indicio, y no es el único, de que "la agricultura vuelve a la agenda internacional".

\subsection{Una última cuestión: ¿se debe proteger a la agricultura?}

Una vez que ha quedado clara la necesidad de las políticas agrícolas, conviene añadir que política agrícola no equivale sin más a proteccionismo agrícola, aunque sea frecuente interpretarlo así. En todo caso estamos ante un debate permanente, y no es bueno concluir estas páginas sin referirnos a él.

Ahora bien, el debate sobre el proteccionismo agrícola debe colocarse en el marco de un debate más genérico: el que se refiere a la alternativa entre mercado puro y mercado con intervención del Estado. Durante las últimas década hemos estado más bien bajo el dominio de los paladines del "todo mercado" y de la liberalización total: ellos critican, no sólo el proteccionismo, sino toda intervención estatal o política activa, y ven en todo eso la causa última de todos los males que afligen a la economía; por eso son decididos defensores de la globalización, en cuanto liberalización del mercado a escala planetaria: "la globalización sólo trae beneficios". Hoy, ante los efectos devastadores de tanto mercado, se vuelve a posturas más equilibradas. El Informe del Banco Mundial recién citado es un exponente inequívoco de este cambio de tendencia, que no es general ni compartido por todos.

En el marco de ese debate más genérico se sitúa el que a nosotros nos interesa más directamente aquí: el relativo al proteccionismo agrícola de la Unión Europea y a sus efectos sobre la agricultura y la alimentación de los países pobres. Los defensores del "todo mercado" lo critican por razones obvias. Pero también 
otras corrientes de pensamiento más intervencionistas e importantes colectivos (movimientos sociales más solidarios) lo critican en nombre de los intereses de los países empobrecidos: afirman que ese proteccionismo agrario europeo perjudica a todo el mundo, menos a los grandes agricultores ${ }^{24}$. Al mismo tiempo, promueven un neo-proteccionismo de las agriculturas de los países en desarrollo.

Frente a esta descalificación sin matices que viene de posiciones ideológicas tan lejanas entre sí, aunque probablemente con móviles profundos diferentes, es deseable llegar a un juicio más ecuánime sobre la Política Agrícola Común (PAC). Ayudará distinguir dos aspectos: la PAC en sí y en sus objetivos directos; sus efectos sobre terceros, y especialmente sobre los países empobrecidos. Aunque es el segundo el que aquí nos interesa, no estará de más decir algo del primero.

La PAC tiene una larga historia ya que nació con la misma Comunidad Económica Europea (en el Tratado de Roma, 1958) y se ha visto obligada a evolucionar al hilo de sus logros y de sus excesos. No podemos entrar en esos detalles. Basta decir que la PAC ha sido el primer mecanismo efectivo de solidaridad supraestatal de la Unión Europea, que ha abierto el camino para otros más amplios instaurados posteriormente. El haber sido escenario de enfrentamientos duros entre gobiernos (con el del Reino Unido, en primer lugar) o entre los sectores más afectados (con el sector agrícola francés) ha hecho difícil su adaptación a las nuevas necesidades surgidas de sus propios resultados. Se puede criticar que el proteccionismo agrícola ha ido demasiado lejos, ha sufrido de una excesiva inercia al cambio, pero ha conseguido un cierto equilibrio de los mercados agrícolas, una aceptable garantía para las rentas de los agricultores y unos precios controlados para los consumidores.

El debate se encona cuando se analizan sus efectos sobre los precios de los alimentos en los mercados mundiales. En aras de la verdad, no existe evidencia empírica que cuantifique estos efectos. Es cierto que las exportaciones subsidiadas y los obstáculos a las importaciones han podido perjudicar, respectivamente, a los consumidores y a los productores de los países en desarrollo. Pero no es menos cierto que los excedentes de la Unión Europea de otros tiempos podrían haber amortiguado la volatilidad de los precios agrícolas en estos últimos meses, si esos excedentes hubieran existido hoy...

En todo caso, el juicio no puede ser unívoco en lo que al proteccionismo se refiere. Hay que ir más al fondo de la cuestión y elaborar propuestas que tengan más en

${ }^{24}$ Cfr. José J. Romero Rodríguez, coordinador (2002) Los efectos de la Política Agraria Europea. Un análisis crítico, Bilbao, Desclée de Brouwer (Colección ETEA). 
cuenta los verdaderos intereses de todos los agricultores, especialmente los pequeños del Norte y los del Sur. Con otras palabras, hace falta una cierta asimetría en el tratamiento de los problemas y no puede usarse la misma vara de medir con los ricos y con los pobres. Parece difícil entrar en sendas duraderas de solución que no pasen por un tratamiento diverso, más proteccionista para los países en desarrollo y menos para los países desarrollados.

Más aún, si los países menos favorecidos del mundo pudieran poner en marcha una política de defensa de sus agriculturas, sin duda intentarían garantizar su propia seguridad alimentaria, muy probablemente establecerían mecanismos de protección frente a los productos del exterior, promulgarían normas de calidad e inocuidad a las que se deberían adaptar sus producciones, garantizarían unos niveles de precios razonables para sus propios productos, establecerían mecanismos de control de excedentes para impedir el derrumbe de los precios en caso de exceso de oferta, y crearían para todo ello un fondo específico destinado a cubrir los gastos derivados de todas esas medidas etc.: es decir, jinventarían una política agraria muy parecida a la que la Unión Europea viene aplicando desde hace casi medio siglo!

\section{A modo de conclusión}

Terminamos el apartado anterior hablando de política agrícola y de proteccionismo. Ambas cosas son necesarias. Pero no son suficientes. Las soluciones duraderas y a largo plazo pasan necesariamente por procesos complejos de planificación nacional y regional (en el sentido de supraestatal), que afecten no sólo a la agricultura.

Si el problema crucial es la seguridad alimentaria, de las que carecen por lo general los pobres, aceptemos que no hay países con hambre en los que no existan grandes desigualdades, y que en los países relativamente igualitarios no suele haber hambre. No hemos querido minusvalorar las responsabilidades de los países desarrollados, pero también queremos subrayar que los países en desarrollo son los últimos responsables de su propio desarrollo. Y esto exige reformas profundas cuyo única base ha de ser un pacto social que permita abordar cuestiones tan decisivas como la consolidación de una administración pública eficaz y transparente, la reforma del sistema fiscal que contribuya al robustecimiento del Estado y permita las inversiones a largo plazo en educación y sanidad (para "empoderar" a las poblaciones más vulnerables), así como en infraestructuras y tecnología. Sin atacar a fondo la desigualdad no se podrá luchar contra el hambre. 
En el Padre nuestro, la oración cristiana por excelencia, suplicamos "Danos hoy nuestro pan de cada día". Sin embargo, la pobreza es mucho más que falta de pan y más allá de la búsqueda afanosa del cotidiano pan material, el desarrollo humano implica -refiriéndonos a la filosofía de Amartya Sen- la ampliación de oportunidades y opciones.

Es en la privación de la vida que la gente puede vivir donde se manifiesta la pobreza. La pobreza puede significar más que la falta de lo que es necesario para el bienestar material. Puede significar la denegación de oportunidades y opciones básicas para el desarrollo humano, vivir una vida larga, sana y disfrutar de un nivel decente de vida, libertad y dignidad, respeto por sí mismo y de los demás ${ }^{25}$.

Ojalá que los responsables de la política y la economía en el mundo de hoy hagan suyas estas palabras, quizás demasiado optimistas, que siguen inmediatamente a las citadas:

Para los encargados de adoptar las decisiones políticas, la pobreza de opciones y oportunidades suele ser más pertinente que la pobreza de ingreso. Centra la atención en las causas de la pobreza y lleva directamente a las estrategias de potenciación y otras medidas encaminadas a realzar las oportunidades de todos ${ }^{26}$.

\footnotetext{
25 Programa de Naciones Unidas para el Desarrollo (PNUD) (1997), Informe sobre desarrollo humano 1997, Washington, 5.

${ }^{26} \mathrm{Ibidem}$.
} 